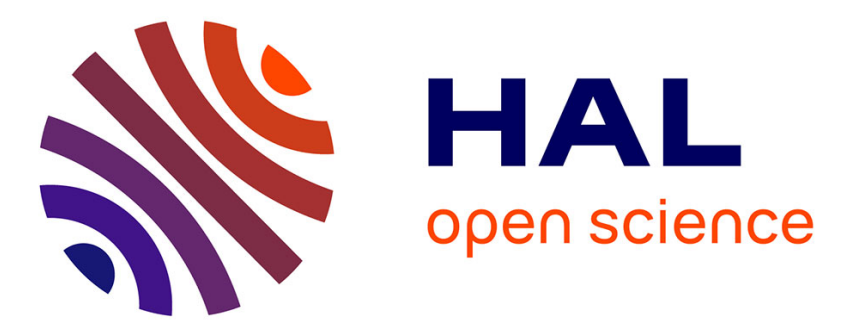

\title{
Coupling Mesoscopic Boltzmann Transport Equation and Macroscopic Heat Diffusion Equation for Multiscale Phonon Heat Conduction
}

\author{
Weizheng Cheng, Ali Alkurdi, P.-O. Chapuis
}

\section{- To cite this version:}

Weizheng Cheng, Ali Alkurdi, P.-O. Chapuis. Coupling Mesoscopic Boltzmann Transport Equation and Macroscopic Heat Diffusion Equation for Multiscale Phonon Heat Conduction. Nanoscale and Microscale Thermophysical Engineering, 2020, 24 (3-4), pp.150-167. 10.1080/15567265.2020.1836095 . hal-03013832

\section{HAL Id: hal-03013832 \\ https://hal.science/hal-03013832}

Submitted on 23 Nov 2020

HAL is a multi-disciplinary open access archive for the deposit and dissemination of scientific research documents, whether they are published or not. The documents may come from teaching and research institutions in France or abroad, or from public or private research centers.
L'archive ouverte pluridisciplinaire HAL, est destinée au dépôt et à la diffusion de documents scientifiques de niveau recherche, publiés ou non, émanant des établissements d'enseignement et de recherche français ou étrangers, des laboratoires publics ou privés. 


\title{
Coupling mesoscopic Boltzmann transport equation and macroscopic heat diffusion equation for multiscale phonon heat conduction
}

\author{
W. Cheng ${ }^{1,2,3}$, A. Alkurdi ${ }^{1}$, P.-O. Chapuis ${ }^{1, *}$ \\ ${ }^{1}$ Univ Lyon, CNRS, INSA-Lyon, Université Claude Bernard Lyon 1, CETHIL UMR5008, F-69621, \\ Villeurbanne, France. \\ ${ }^{2}$ École Centrale de Nantes, Nantes, France \\ ${ }^{3}$ School of Materials Science and Engineering, Shanghai Jiao Tong University, Shanghai 200240, PR \\ China \\ Keywords: Multiscale, Phonon heat conduction, Boltzmann equation, Discrete Ordinate Method \\ * Corresponding author: Pierre-Olivier Chapuis (olivier.chapuis@insa-lyon.fr)
}

\begin{abstract}
Phonon heat conduction has to be described by the Boltzmann transport equation (BTE) when sizes or sources are comparable to or smaller than the phonon mean free paths (MFPs). When domains much larger than MFPs are to be treated or when regions with large and small MFPs coexist, the computation time associated with full BTE treatment becomes large, calling for a multiscale strategy to describe the total domain and decreasing the computation time. Here, we describe an iterative method to couple the BTE, under the Equation of Phonon Radiative Transfer approximation solved by means of the deterministic Discrete Ordinate Method, to a Finite-Element Modelling commercial solver of the heat equation. Small-size elements are embedded in domains where the BTE is solved, and the BTE domains are connected to a domain where large-size elements are located and where the heat equation is applied. It is found that an overlapping zone between the two types of domains is required for convergence, and the accuracy is analysed as a function of the size of the BTE domain. Conditions for fast convergence are discussed, leading to the computation time being divided by more than five on a study case in 2D Cartesian geometry. The simple method could be generalized to other types of solvers of the Boltzmann and heat equations.
\end{abstract}




\section{Introduction and goals}

Heat conduction at nanoscale diverges from usual macroscopic heat diffusion in crystalline solids, as has been shown in the last decades both theoretically, numerically and experimentally. A key reason is that energy carriers do not undergo as many collisions at small scale due to their finite mean free path ${ }^{1-}$ ${ }^{4}$. For phonons, the average mean free path (MFP) is around $180 \mathrm{~nm}$ in silicon, with a distribution spanning from nanometers to tens of micrometers ${ }^{5,6}$. In order to describe accurately phonon transport, the Boltzmann transport equation (BTE), an equation of conservation of the density of energy carriers, is the tool of choice. The BTE for phonons was highlighted by Debye and Peierls and has been used successfully since that time. Since the phase space distribution function depends on 7 variables (the space variables, two variables for the direction, one for the frequency and one for time) in general, and 6 in the steady state, numerical solutions can be long to be obtained if the spatial domain becomes large, i.e. much larger than the MFP. There are two main types of methods than can be used for solving the BTE: stochastic methods, such as those involving Monte Carlo (MC) sampling of the dispersion curves or free paths ${ }^{7-9}$, and deterministic methods ${ }^{10}$. Among the last category, the Discrete Ordinate Method (DOM) is a popular method ${ }^{6,11-14}$, especially in the thermal radiation community where it has been developed since decades. The Radiative Transfer Equation (RTE) is an integrated version of the BTE, where the distribution function is replaced by the spectral radiance. The tools developed for the RTE can be applied for phonons without much difficulty, leading to the so-called Equation of Phonon Radiative Transfer (EPRT), where "radiative" is here meant as "ballistic" 15 . One has mostly to pay attention to the dispersion of phonons. Note that considerable body of work was realized in this field and that not all contributions can be mentioned here.

Due to the large number of variables involved in the BTE and the large aspect ratios in the configurations to be considered, that can span more than 5 orders of magnitude, very lengthy computation times, when the problem is tractable in practice, are often observed. In order to circumvent such issue, different solutions can be envisaged ${ }^{12,16-22}$. Some of them involve splitting the phonon spectrum in order to solve the full BTE only for long MFPs ${ }^{12,19,20}$. Such strategy can be implemented into the whole computational domains. This can mean that long MFPs are still treated with BTEs far from the regions where nanoscale heat dissipation takes place, and there is still room for improvement as a consequence. The ballisticdiffusive equations ${ }^{16,23}$ and associated improvements ${ }^{12}$ are also an option, based on well-known methods of the thermal radiation community ${ }^{24}$ treating the interaction with walls of the domains more accurately. The physics is sometimes complex and not everyone is able to deal with such frameworks, so there is a lack for simple tools. Here, we introduce a coupling between the BTE for phonons, which solves the phonon transport accurately at small scale, and the heat equation (HE), which is sufficient at large scale. Similar approaches have been addressed by Vallbahaneni et al. ${ }^{21}$ and Li et al. ${ }^{22}$, but in an effective onedimensional heat conduction configuration involving further complexity due to the stochastic nature of the Monte Carlo approach ${ }^{22}$ or by including thermal boundaries between materials which eliminate some 
complications as will be shown ${ }^{21}$. The present article investigates the coupling conditions in 2D Cartesian geometry between the phonon BTE solved by DOM and the heat equation solved by the FiniteElement Method (FEM). Here, the first code is an in-house DOM code ${ }^{13,25}$ developed based on the work of Lemmonier and colleagues for thermal radiation, but other codes could certainly be considered instead. The FEM tool is commercial (COMSOL). An iterative method where boundary temperature and flux are exchanged between sub-domains where each equation is solved is introduced. Various difficulties are underlined, and it is found that an overlap between the two sub-domains allows converging toward the proper temperature and flux fields. The size of the DOM sub-domain required for accurate fields is investigated, as well as the computation time. It is found that the method can decrease the computation time by a factor larger than $\sim 5$ for computation over sub-millimeter sizes and makes it tractable to deal with domains of arbitrary size involving small scale phonon transport features. Note that this method is not based on the use of effective thermal conductivity or other effective parameters in FEM simulations, which can only provide approximate solutions. While the single MFP approximation is considered here for practical purpose, the method can be extended to full MFP distribution BTE deterministic framework under certain conditions. We also anticipate that it could be a basis for the coupling of MC methods with solvers of the heat equations.

The paper is organized as follows. We first explain how the local temperature is computed in the frame of the EPRT approximation. We then remind few key points in the 1D configuration and indicate the 2D Cartesian studied configuration. We then explain the different strategies tested for the coupling, and finally present the main outputs of the work.

\section{Equation of Phonon Radiative Transfer with an average mean free path}

\section{II.A. Boltzmann transport equation for phonons}

Phonons, quantized lattice vibrations, are known as one of the main energy carriers in solids and transport energy by interaction among them. When the mean free path of travelling phonons is much larger than or comparable to dimension of the domain that is studied, phonons have few interaction with each other and local thermodynamic equilibrium does no longer exist. Heat transport is dominated by ballistic heat transfer and the Fourier law loses its validity.

The BTE is a successful tool for describing (quasi-)particle, therefore incoherent transport in both rarefied and dense media, and thus can be applied for phonons (see e.g. ${ }^{1-4}$ ). In a system without external applied force, the equation is written in a simplified form under the relaxation time approximation:

$$
\frac{\partial f}{\partial \mathrm{t}}+\boldsymbol{v}_{g, p} \cdot \nabla f=\frac{f^{0}-f}{\tau_{\omega}}
$$

where $f(t, \boldsymbol{r}, \boldsymbol{s}, \omega)$ is the distribution of phonons and depends on time $t$, position $\boldsymbol{r}$, emitted direction $\boldsymbol{s}$ and angular frequency $\omega, f_{0}$ is the equilibrium distribution function of phonons, $\boldsymbol{v}_{g}$ is the phonon group velocity depending on the phonon polarization $p$ (one longitudinal acoustic (LA) mode and two 
transverse acoustic (TA) modes are considered here), and $\tau_{\omega}$ is the frequency-dependent relaxation time of phonon-scattering processes. Note that the polarization dependence of $f$ is omitted for the sake of brevity. The position $\boldsymbol{r}$ has three components while the direction vector $\boldsymbol{s}$ has two, namely the polar angle $\theta$ and azimuthal angle $\varphi$. The left side of Eq. (1) represents changes in distribution function caused by motion, which deviates phonons from equilibrium state in phase space, and the right side represents changes caused by the collisions (interactions) of phonons, which usually tend to restore the phonon local equilibrium. Relaxation time approximation allows generalising scattering events by considering in an average way all collisions undergone by a group of phonons. It is well-known that phonons are quasi-particles obeying the Bose-Einstein statistics,

$$
f^{0}=\frac{1}{\mathrm{e}^{\frac{\hbar \omega}{k_{B}}}-1},
$$

where $\hbar$ is the reduced Planck constant, $k_{B}$ is the Boltzmann constant and $T$ is temperature. Similarly to the photon radiance (intensity) in the RTE, the phonon spectral radiance (intensity) can be defined as

$$
I_{\omega, p}=I(t, \boldsymbol{r}, \boldsymbol{s}, \omega)=\boldsymbol{v}_{g, p} \cdot \hbar \omega \cdot f \cdot \frac{D(\omega)}{4 \pi}
$$

where $D(\omega)$ is the phonon density of states per unit volume. Combining Eq. (1) and (3) and introducing the mean free path of phonon $\Lambda_{\omega}=\tau_{\omega}\left|\boldsymbol{v}_{g, p}\right|$, the BTE can be written in the phonon intensity form as

$$
\frac{1}{\left|v_{g, p}\right|} \frac{\partial I_{\omega, p}}{\partial \mathrm{t}}+\boldsymbol{s} \cdot \boldsymbol{\nabla} I_{\omega, p}=\frac{1}{\Lambda_{\omega}}\left(I_{\omega, p}^{0}-I_{\omega, p}\right)
$$

where $I_{\omega, p}^{0}=I_{\omega, p}$ for $f^{0}$. Due to the similarity with the RTE, Eq. (4) is called the equation of phonon radiative transport (EPRT). Solving an equation over the density of quasiparticles (Eq. (1)) or over the density of energy (Eq. (5)) in the phase space leads to the same result and the same computational cost. By using normalized time $t^{*}=t / \tau_{\omega}$ and $\boldsymbol{r}^{*}=\boldsymbol{r} / L$, where $\tau_{\omega}$ is the average relaxation time of collision of phonon for angular frequency $\omega$ and $L$ is the characteristic scale of the treated configuration (therefore $\boldsymbol{\nabla}^{*}=\frac{\partial}{\partial \boldsymbol{r}^{*}}=L \boldsymbol{\nabla}$ ), it is easily deduced that

$$
\frac{\partial I_{\omega, p}}{\partial t^{*}}+\mathrm{Kn}_{\omega, p} \cdot \boldsymbol{s} \cdot \boldsymbol{\nabla}^{*} I_{\omega, p}=I_{\omega, p}^{0}-I_{\omega, p}
$$

where the dimensionless Knudsen number, i.e. the normalized mean free path (MFP), is defined as $\mathrm{Kn}_{\omega, p}=\Lambda_{\omega, p} / L$. It allows determining whether heat transport is dominated by diffusive or ballistic heat transfer. Previous work showed that when $\mathrm{Kn}<0.02$ heat transfer is diffusive and the Fourier law accurately predicts flux and temperature fields, showing a difference lower than $2 \%$ compared to the predictions of the EPRT in 1D model. By solving Eq. (4), phonon spectral radiance at position $\boldsymbol{r}$ and in direction $\boldsymbol{s}$ can be firstly found out. Then, by integrating on solid angle and frequency and by summing up phonon modes, internal energy $e$ and heat flux $\boldsymbol{q}$ can be formulated as

$$
e(t, r)=\sum_{p} \int_{0}^{\omega_{\max }} \int_{4 \pi} \frac{I_{\omega, p}(t, r, s)}{\boldsymbol{v}_{g, p}} d \Omega d \omega
$$




$$
\boldsymbol{q}(t, \boldsymbol{r})=\sum_{p} \int_{0}^{\omega_{\max }} \int_{4 \pi} I_{\omega, p}(t, \boldsymbol{r}, \boldsymbol{s}) \boldsymbol{s} d \Omega d \omega
$$

where $d \Omega=\mathrm{d} \theta \cdot \sin \theta \cdot d \varphi$ is the elemental solid angle and $\omega_{\max }$ is the cut-off frequency depending on the phonon polarization. Temperature field at equilibrium can be found from Eq. (2) (note $\boldsymbol{q}\left(I^{0}\right)=\mathbf{0}$ ). A full Boltzmann treatment of phonon transport requires solving Eqs. (5-7). It can result in the local energy distribution deviating from equilibrium, i.e. being direction-dependent or having its intensity following a different statistics than the equilibrium one. It is often useful to plot 'kinetic' temperature distributions from the density of energy $e(t, r)$ by determining the equilibrium temperature $T$ so that

$$
\sum_{p} \int_{0}^{\omega_{\max }} \int_{4 \pi} \frac{I_{\omega, p}^{0}(T)}{v_{g, p}} d \Omega d \omega=e(t, \boldsymbol{r}) .
$$

It is only a measure of the local energy, and should not be used to derive other thermodynamics quantities.

\section{II.B. Average mean free paths}

Integrating Eq. (5) over frequency and summing over the polarizations leads to the following equation:

$$
\frac{\partial I}{\partial t^{*}}+\left(\sum_{p} \int_{0}^{\omega_{\max }} \mathrm{Kn}_{\omega, p} \cdot \boldsymbol{s} \cdot \boldsymbol{\nabla}^{*} I_{\omega, p} d \omega\right)=I^{0}-I,
$$

where $I=\sum_{p} \int_{0}^{\omega_{\max }} I_{\omega, p} d \omega$ is the phonon total radiance and therefore $I^{0}=\sum_{p} \int_{0}^{\omega_{\max }} I_{\omega, p}^{0} d \omega$. This equation can be further simplified by introducing an average mean free path $\bar{\Lambda}$ and suggests to rewrite:

$$
\frac{\partial I}{\partial t^{*}}+\overline{\mathrm{Kn}} \cdot \boldsymbol{s} \cdot \nabla^{*} I_{\omega, p}=I^{0}-I
$$

where $\overline{\mathrm{Kn}}=\bar{\Lambda} / L$. Eq. (9) is an EPRT for the total radiance. If Eq. (10) is sufficient, this means that a single equation is to be solved, instead a large number depending on the spectral discretization in Eq. (5). Let us now comment on the derivation of Eq. (10), which is not at all straightforward despite its apparent intuitiveness. First, in the diffusive regime, the local distribution is only weakly distorted from equilibrium, and it is usual to write $\nabla I_{\omega, p}=\frac{\mathrm{d} I_{\omega, p}^{0}}{\mathrm{~d} T} \nabla T$. In this case one can equate Eq. (9) and Eq. (10), and this leads to an expression for the averaged mean free path:

$$
\bar{\Lambda}_{0}=\frac{1}{\frac{\mathrm{d} I^{0}}{\mathrm{~d} T}} \sum_{p} \int_{0}^{\omega_{\max }} \frac{\mathrm{d} I_{\omega, p}^{0}}{\mathrm{~d} T} \Lambda_{\omega, p} d \omega
$$

This expression is equal to the average mean free path determined from thermal conductivity $(k=$

$$
\begin{aligned}
\left.\sum_{p} \int_{0}^{\omega_{\max }} \int_{4 \pi} \frac{\mathrm{d} I_{\omega, p}^{0}}{\mathrm{~d} T} \Lambda_{\omega, p} \cos ^{2} \theta d \Omega d \omega\right): \\
\bar{\Lambda}_{0}=\frac{\sum_{p} \int_{0}^{\omega_{\max }} \int_{4 \pi} \frac{\mathrm{d} I_{\omega, p}^{0}}{\mathrm{~d} T} \Lambda_{\omega, p} \cos ^{2} \theta d \Omega d \omega}{\sum_{p} \int_{0}^{\omega_{\max }} \int_{4 \pi} \frac{\mathrm{d} I_{\omega, p}^{0}}{\mathrm{~d} T} \cos ^{2} \theta d \Omega d \omega}=\frac{k}{\frac{1}{3}<c v>} .
\end{aligned}
$$

where $\langle c v\rangle=\sum_{p} \int_{0}^{\omega_{\max }} \int_{4 \pi} \frac{d I_{\omega, p}^{0}}{d T} d \Omega d \omega=\sum_{p} \int_{0}^{\omega_{\max }} c_{p}(\omega) v_{g, p}(\omega) d \omega$ is an integral over acoustic modes, involving the volumetric heat capacity associated to acoustic modes and the mean group velocity of phonons $v$. For silicon, one finds $\bar{\Lambda}_{0}=183 \mathrm{~nm}$ at $300 \mathrm{~K}$ for $k=148 \mathrm{~W} \cdot \mathrm{m}^{-1} \cdot \mathrm{K}^{-1}$. In this regime, 
considering the average mean free path in Eq. (5) or the whole distribution does not make any difference, and it is of course easier to solve Eq. (10). However, when far from the diffusive regime ( $\overline{\mathrm{Kn}} \gg 0.01)$, the previous way of deriving Eq. (10) is not possible in principle. In the particular cases where the temperature-dependence of the spectral mean free paths can be neglected or where the mean free path distribution is assumed homogeneous in the whole domain, one can obtain the following expression for the averaged mean free path:

$$
\bar{\Lambda}=\frac{1}{I} \cdot \sum_{p} \int_{0}^{\omega_{\max }} \Lambda_{\omega, p} \cdot I_{\omega, p} d \omega .
$$

In the ballistic regime, the local phonon radiance deviates more strongly from equilibrium, and, as a result, the radiances in Eq. (13) are not equilibrium ones. This means that the averaged mean free path is different to that of Eqs. $(11,12)$. It can be angle-dependent, and hemispherical (two values depending on direction) in the ballistic limit in a 1D configuration. However, we will solve Eq. (10) and keep Eq. (11) (around room temperature) in the following sections, for the sake of simplicity. Currently, the spectral mean free paths considered within BTE are those determined at equilibrium, so there is anyway probably some variation when in the ballistic regime. Addressing this issue is much beyond the scope of this article.

Note that simplified version of Eqs. $(9,11)$, where the integrals cover the whole frequency range (no cutoff) and where possibly a single (averaged) polarization is used, were sometimes considered in the past, as this allows to formulate an equation over $I=\int_{0}^{\infty} I_{\omega} d \omega$ with simple analytical expressions in a fashion parallel to the $I^{0}=\sigma T^{4} / \pi$ case of photons ${ }^{15,20,22}$. Such approach can lead to plausible temperature profiles at room temperature, but leads unfortunately to very inaccurate fluxes. This approach should therefore be limited to low temperature, where the ratio of the Debye frequency to the Wien one is large.

\section{II.C. Brief reminder on the Discrete Ordinate Method}

In this work, we solve the EPRT by means of the Discrete Ordinate Method (DOM) ${ }^{25,26}$. The main idea of DOM is to discretize the angular space in a finite number of directions. The phonon intensity is computed in each direction, and the integration along the solid angle is replaced by a discrete sum over the discretized angles with weights. Since all directions of phonon radiation are treated by DOM, it can be used in particular for non-isotropic boundary conditions like specular reflection or intermediate ones ${ }^{20}$. The EPRT in a steady-state 2D problem, where the first term of Eq. (10) vanishes, is first discretized as a function of the solid angle as follows:

$$
\mu_{m} \frac{d I_{m}}{d x}+\eta_{m} \frac{d I_{m}}{d y}=\frac{I_{m}^{0}-I_{m}}{\overline{\mathrm{Kn}}}
$$

where $\mu_{m}, \eta_{m}$ are respectively the $x$ - and $y$-projections of $\boldsymbol{s}_{m}$, where $m$ is the index associated to one direction in the whole discretized angular domain, and $I_{m}$ is phonon intensity in direction $\boldsymbol{s}_{m}$. The 
number of directions is selected to fulfil certain symmetries of the problem and $m \in[1, N(N+2)]$ for a $S_{N}$ discretization scheme. The reader is referred to Refs. (19-20) for more extensive details on $S_{N}$. With $w_{m}$ representing the weight of the discretized elemental solid angle in direction $\boldsymbol{s}_{m}$, the integration along the solid angle is transformed into the discrete form and so are internal energy and heat flux:

$$
\begin{gathered}
\sum_{m=1}^{N(N+2)} w_{m}=\int_{4 \pi} d \Omega \\
e=\sum_{p} \int_{0}^{\omega_{\max }} \sum_{m=1}^{N(N+2)} \frac{w_{m} I_{\omega, p}\left(t, \boldsymbol{r}, \boldsymbol{s}_{m}\right)}{\boldsymbol{v}_{g, p}} d \Omega \\
\boldsymbol{q}=\sum_{p} \int_{0}^{\omega_{\max }} \sum_{m=1}^{N(N+2)} w_{m} I_{\omega, p}\left(t, \boldsymbol{r}, \boldsymbol{s}_{m}\right) \boldsymbol{s}_{m} d \Omega
\end{gathered}
$$

One issue of the method is that some ray effects, i.e.an overestimation of certain directions in the ballistic regime, may appear when $N$ is too low. We have found that $N=12$ allows to obtain relatively smooth angular patterns in 2D with moderate computational $\cos ^{27}$, and such discretization scheme is used in the following. $S_{6}$ is clearly insufficient, and if computational cost can be extended, $S_{20}$ can be considered. Note that the CADOM approach ${ }^{12}$, not performed here, helps in decreasing the impact of the ray effect. In the present study, the spatial domain is discretized by regular elements in Cartesian geometry and a Finite Volume Method is implemented (see Ref. 19 for more details).

\section{Key features of the transition from diffusive to ballistic transfer in a 1D geometry}

(b)

(a)

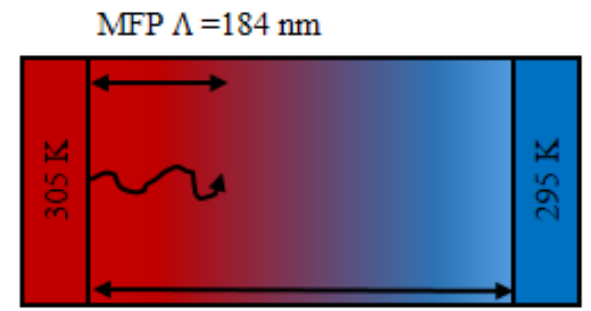

Domain size $\mathrm{L}=40 \sim 10000 \mathrm{~nm}$

$\mathrm{Kn}=\Lambda / \mathrm{L}=5 \sim 0.02$

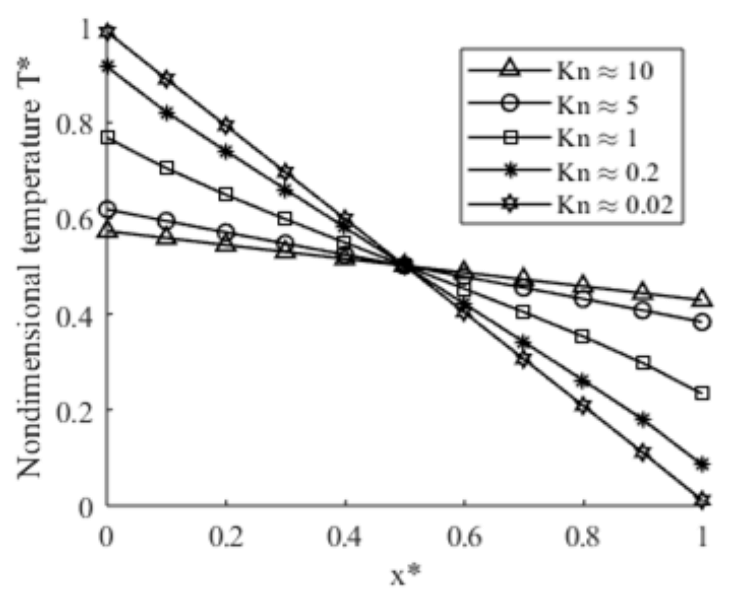


(c)

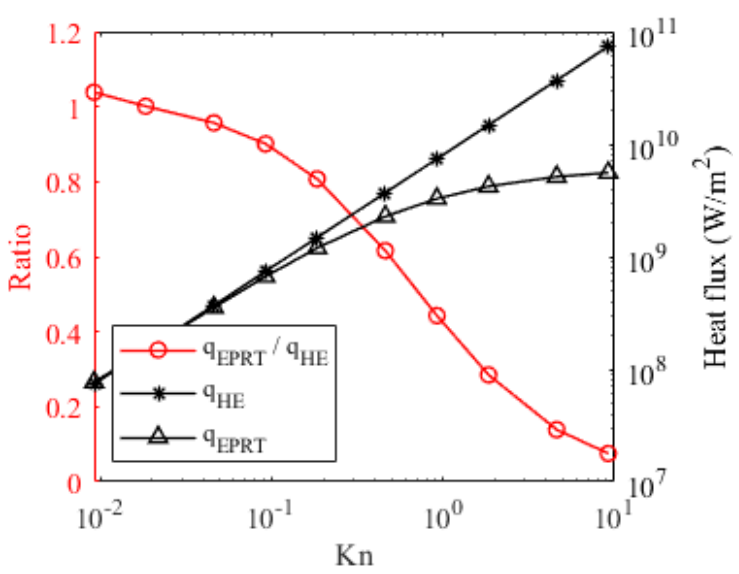

(d)

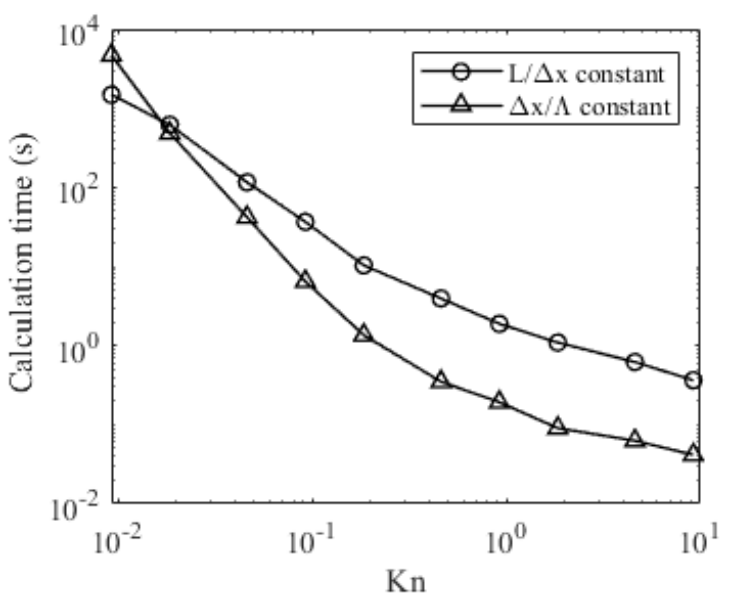

Figure 1. Simulation with EPRT by DOM in a 1D problem. (a) 1D configuration and parameters, with the tested domain size varying from microscale to macroscale; (b) Normalized temperature profile with various tested domain sizes, i.e. $\mathrm{Kn}$; (c) Heat flux normalized by the corresponding predictions from the Fourier law as a function of the nondimensioned length (left scale) and absolute values from EPRT and HE for the same domain sizes; (d) Calculation times as a function of Kn. Two cases are shown: fixed number of mesh elements (circles) and fixed mesh element size $\Delta x$ (triangles).

We first start by few reminders on the transition from the diffusive to the ballistic regime in a simple configuration ${ }^{28}$. Based on the previous work by Volz et al. ${ }^{13}$, the DOM code for solving the EPRT is applied to a 1D domain. Note that the current DOM solver of COMSOL (used e.g. in Ref. [6]) was found to be very slow in comparison to our solver ${ }^{27}$ and, in addition, may not be not straightforward to accommodate to the need for considering the right spectral range for the phonon intensity. This is unfortunate as this could have allowed to implement easily the coupling strategy in a fully-commercial widespread software. Boundary conditions are shown in Fig. 1(a). Bulk silicon is chosen for all simulations, with the average MFP of phonon set equal to $180 \mathrm{~nm}$. In order to characterize properties of heat transfer from the ballistic to the diffusive regime, the length of the 1D region ranges from $40 \mathrm{~nm}$ to $10 \mu \mathrm{m}$, which means that $\mathrm{Kn}$ (the overline denoting the averaging is omitted in the following) varies accordingly from 5 to 0.02 . DOM calculations were considered as converged once relative difference of phonon intensity between current iteration and previous one was less than $10^{-18}$. It is worth mentioning that when ballistic conduction dominates the critical value of the relative difference above can be relaxed to $10^{-12}$, resulting in a difference of temperature less than $10^{-6} \mathrm{~K}$. However, for quasi-ballistic and diffusive problem, the critical value should be stricter and no more than $10^{-15}$ in order to keep the same accuracy. 
Fig. 1(b) shows the normalized temperature $T^{*}=\frac{T\left(x^{*}\right)-T_{\min }}{T_{\max }-T_{\min }}$ profile for various medium lengths while number of elements in the mesh is kept constant and is equal to 1000. The solution of the EPRT has little difference with Fourier's law prediction under the condition $\mathrm{Kn}<0.02$, confirming that heat transfer is almost mediated by diffusive transport for large sizes. We just note a slight deviation to ratio 1 at small $\mathrm{Kn}$ in Fig. 1(c) due to the limited discretization scheme $S_{12}$ selected as a good compromise for all calculations. Decreasing the domain length, the transition towards ballistic transport progressively takes place, leading to temperature jumps at the contacts with the thermostats. In the ballistic limit, the temperature profile is flat. It is interesting to analyze the deviation to the Fourier prediction as a function of medium length. Fourier's law $\boldsymbol{q}_{\text {Fourier }}=-k \boldsymbol{\nabla} T$ tells us that in 1D $q=k \frac{T_{\max }-T_{\min }}{L}$. We normalize the heat flux found by the EPRT by this value. It is observed that, compared with diffusive heat transfer, ballistic transport is not able to efficiently transfer heat as a consequence of the lack of phonon collisions. Besides, as an important feature for the following, calculation time of the EPRT is displayed in Fig. 1(d). By keeping the number of elements in the mesh as constant, the slope varies only in a limited way, indicating that computational time increases exponentially from seconds to hours when decreasing Kn. That is because the EPRT describes changes due to collisions and there are much more collisions in the diffusive regime $(\mathrm{Kn} \ll 1)$ than in the ballistic one $(\mathrm{Kn} \gg 1)$. In the other case where the size of mesh element is kept constant, which seems more accurate, the computational time increases much faster when Kn decreases because the quantity of mesh elements increases on top of the previous reason. Such test indicates that larger-dimension domains (2D or 3D) could lead to very-large and probably intractable computation time in the case of complex configurations, reflecting the necessity of finding alternative strategies than full-BTE computation. Coupling the EPRT and the heat equation based on Fourier's law is the option studied in the following.

\section{Heat dissipation from wire-on-substrate configuration}




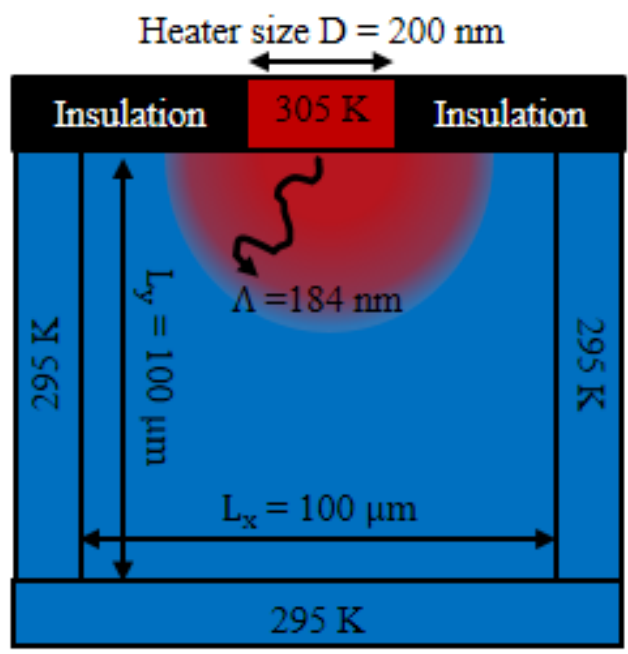

Figure 2. 2D Cartesian wire-on-substrate configuration under study.

The tested configuration is reminiscent of the nanowire of rectangular cross-section deposited on top of a silicon substrate. This configuration has been considered in particular in the works by Mazumder et al. ${ }^{11}$ and experimentally tested by groups in Boulder ${ }^{29}$, Lyon $^{30}$, Purdue $^{31}$, Caltech $^{32}$ and MIT ${ }^{33}$. The wire undergoes either Joule self-heating due to the transverse flow of electrical current or is simply heated remotely in non-contact way as it absorbs partly light from an optical beam at a wavelength where the substrate is transparent. Here, we consider only steady state, in contrast to some of the experimental works. The wire is treated as fixed-temperature segment of length $l$ on top of the domain. The size of the segment was varied, but we will mostly report the $l=200 \mathrm{~nm}$ case. This is close to the average MFP of silicon at room temperature, therefore the Knudsen number associated to the heat source is $\mathrm{Kn} \sim 1$, thus ballistic conduction occurs around the thermal source. The domain is $100 \mu \mathrm{m}$ large, but due to the adaptive mesh of current FEM solvers, for which the number of mesh elements does not scale steeply with the size, the cost of increasing the domain would not be important. Far from the heater, for instance at a distance larger than 50 times the MFP ( $10 \mu \mathrm{m}$ in our case), thermal conduction is dominated by diffusive conduction. Hence, the whole domain can be divided into two parts: a region where accounting for ballistic dissipation is required and another region where heat diffusion is sufficient.

\section{IV.B. Estimation of heat dissipation}

Heat dissipation from the source can be estimated based on values of the thermal resistance to a large heat bath. This does not provide the temperature or flux distributions inside the domain, but can still be useful. For very small $\mathrm{Kn}$, i.e. large sources, the dissipation can be treated analytically. To provide an idea, let us consider that the heater is a half circle of radius $a$ and the total domain a half disc of radius $b$. In this cylindrical configuration, we immediately find that the thermal resistance is $R=\frac{1}{\pi k} \ln \frac{b}{a}$, where 
(a)

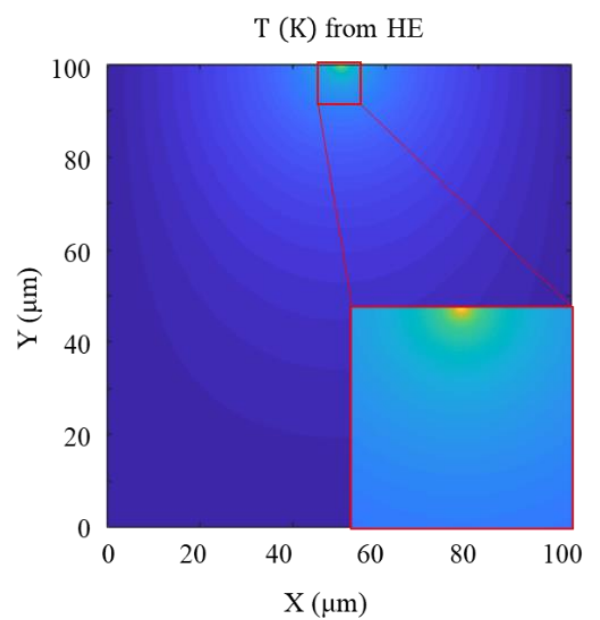

(b)
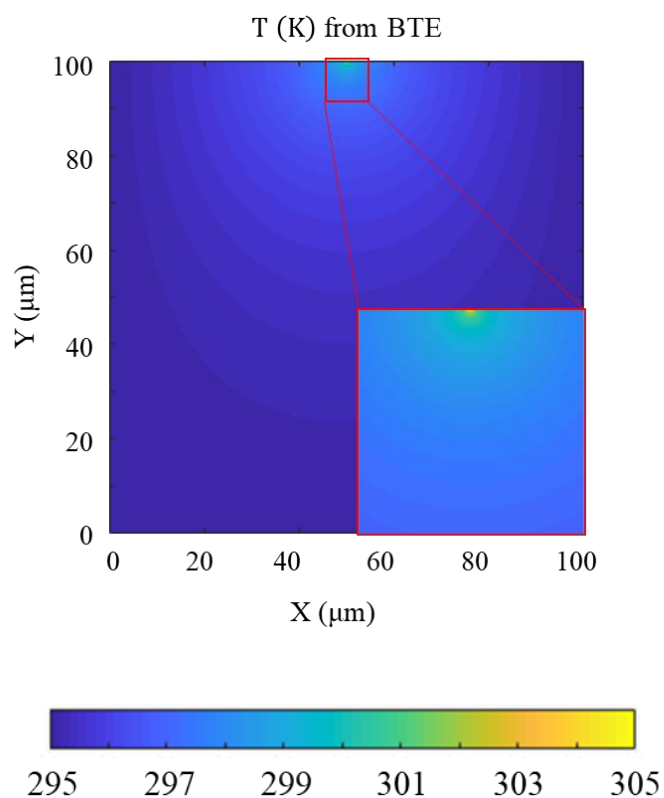

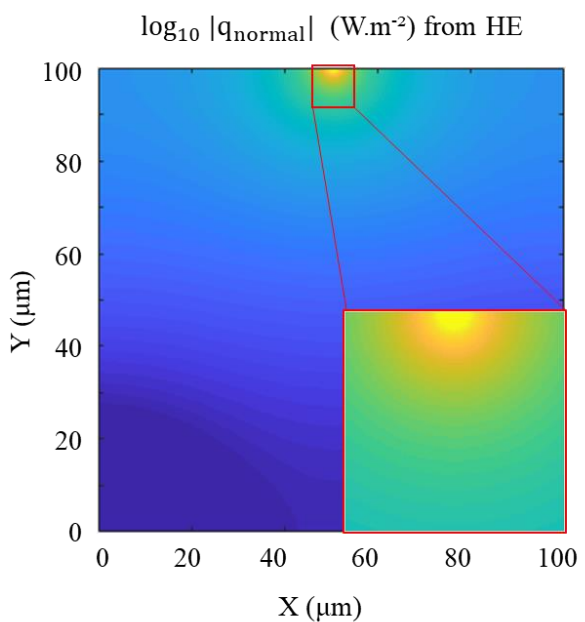

(d)

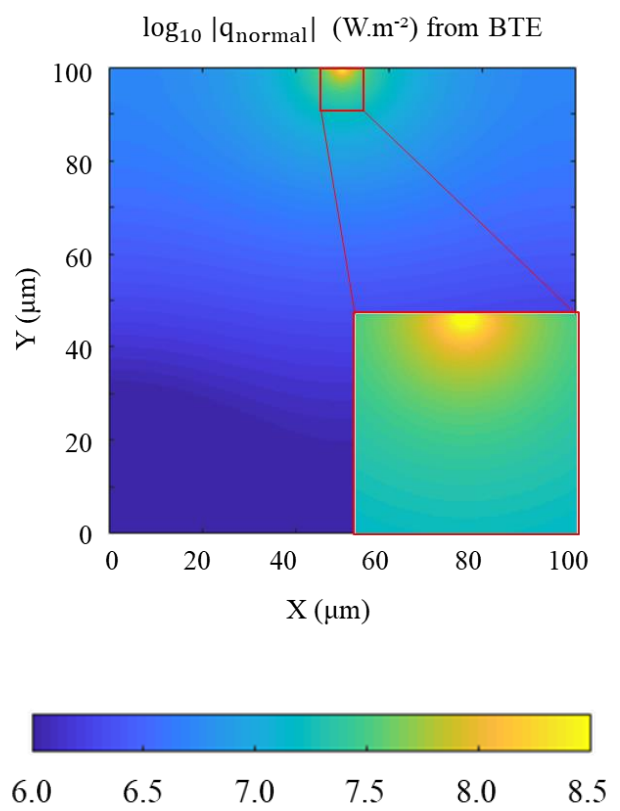

Figure 3. Reference solutions obtained from the heat equation $(\mathrm{a}, \mathrm{c})$ and from the BTE-EPRT solver (b,d). (a,b) Temperature fields. (c,d) Heat flux fields.

$k$ is the thermal conductivity. A logarithmic dependence on source and domain sizes is observed. The Cartesian geometry can also be treated analytically for the case of a finite thickness $t$ and infinite domain width, and one finds a thermal resistance per unit length:

$$
R_{d i f}=\frac{1}{\pi k}\left[\ln \left(\frac{b}{t}\right)-\beta\right],
$$


where $\beta=1.0484^{34}$. Another limit is the very large Kn case, where the dissipation is fully ballistic and the heat transfer is fully dominated by the ballistic resistance per unit length $R_{b a l}$ that can be computed analytically:

$$
R_{b a l}=\frac{4}{<c \quad v>b}
$$

In the transition regime where $b \sim \Lambda$, the resistance is often treated with the Wexler approximation ${ }^{35}$, a Matthiessen rule between the diffusive and ballistic cases $R_{d i f}+R_{b a l}$. Such approximation is known to depart by few tens of per cents from the exact solution in this intermediate regime, so a full numerical treatment involving the BTE is to be applied for quantitative results. As a side comment, let us recall the reason why the Wexler approximation is not so far from the exact result. Heat flows from the constriction towards the bath, so if the source characteristic width is sub-MFP the total thermal resistance is the sum of a ballistic resistance and a diffusive one, the latter being associated with a region away

from the source. It can be estimated that this region starts for a radial distance $b_{\text {diff }} \approx K \Lambda$, where $K=O(1)$ is a geometrical parameter larger than 1. If the source size is much larger than $\Lambda$, then only Eq. (18) applies. In the end, a weighted average of $R_{\text {dif }}$ and $R_{b a l}$ is sufficient to describe the total thermal resistance, with weights being functions of $(t, b)$ and equal to unity for each resistance asymptotically. Here, it can be shown that the thermal total resistance is dominated by $R_{d i f}$.

In order to obtain the full temperature and flux fields a full BTE resolution, involving the coupling framework in the case of large values $t$, is anyway required. Figs. 3 provide reference results obtained either with the heat equation (HE) or with the BTE in the EPRT approximation (computation time discussed later in Tab. 1). It can be seen that for this particular configuration the correct temperature field (BTE) is close to that obtained with the HE when away from the hot source, in agreement with the suggestion that the thermal total resistance is dominated by $R_{\text {dif }}$. Nevertheless, the local temperature is lower than that predicted by the HE just below the heat source. In addition, the flux fields appear to be very similar in the two cases, as dictated be the conservation of energy, albeit small difference can be observed. The reader is referred to previous studies for detailed analyses of this configuration ${ }^{11,31}$.

\section{Coupling conditions}

\section{V.A. Initial idea of the coupling process}

The coupling scheme to be tested is represented in Fig. 4. With the aim of saving numerical calculation time, the whole domain is initially separated into two regions as shown in Fig. 5(a). The BTE subdomain, which is around the heat source, is associated to a Knudsen number involving its size $(\sim 10 \mu \mathrm{m}$ in this example): $\mathrm{Kn}_{\mathrm{x}}=\mathrm{Kn}_{\mathrm{y}}=0.02$. DOM is applied in this sub-domain. The rest of the medium is the HE sub-domain, where the Fourier law can be applied without causing much error by using a FEM 
solver (commercial COMSOL tool used in our case). The working principle of coupling BTE and HE, displayed in Fig. 4(a), is initially as follows. In the zeroth step, the whole domain is initialized with HE, which allows getting initial values of temperature $T_{b}$ and flux $\boldsymbol{q}_{b} \cdot \boldsymbol{n}=q_{b n}$ at the BTE-HE boundary, where $\boldsymbol{n}$ is the unit vector normal to the boundary. Then, the first step starts when the BTE is solved on its sub-domain by DOM (EPRT code programmed in Fortran) with boundary condition $T_{b}$ (in conjunction with the other conditions at the external boundaries which are fixed), so that temperature and heat flux fields are obtained within the BTE sub-domain.

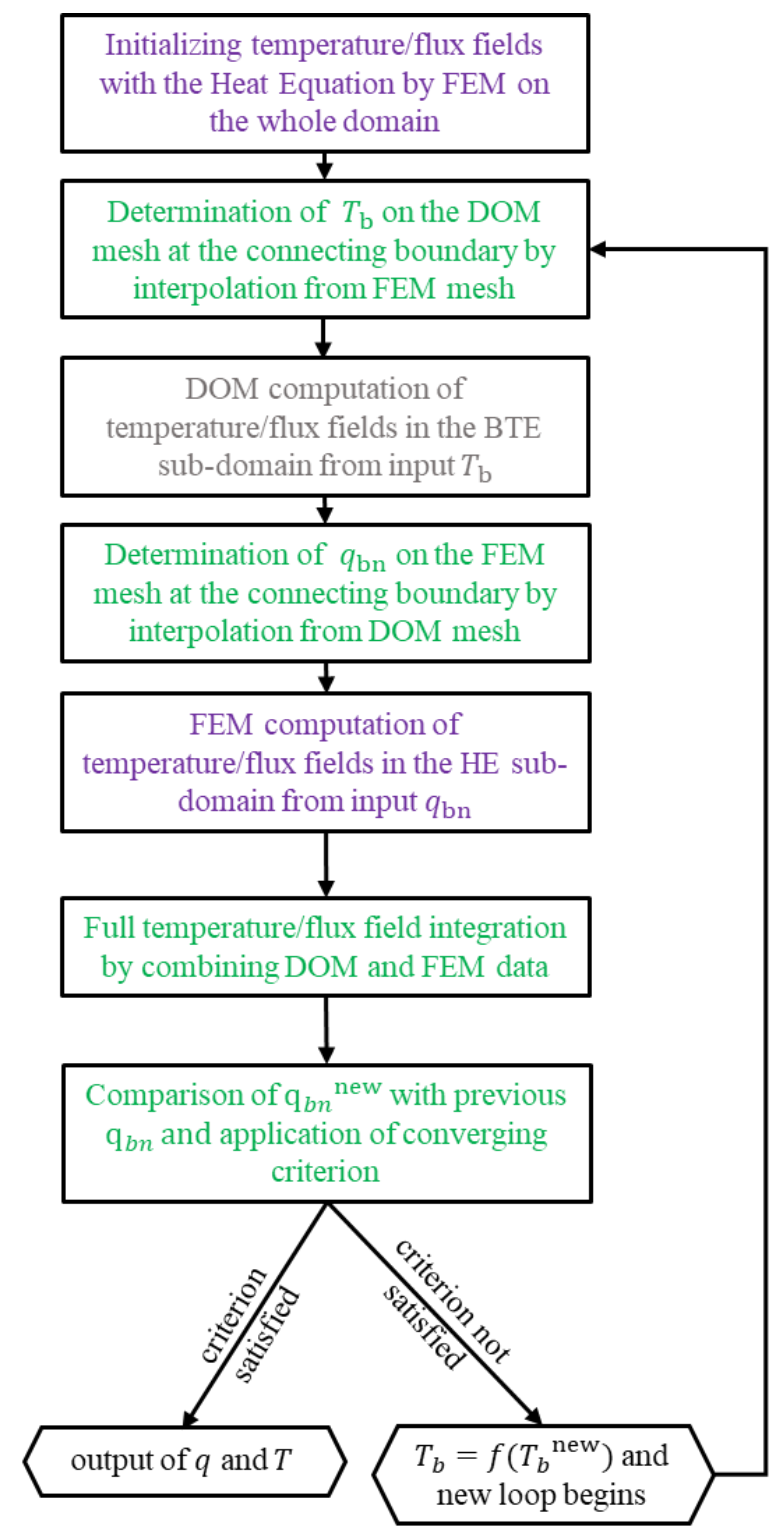

Figure 4. Coupling scheme involving two sub-domains where the Heat Equation and the Boltzmann Transport Equation in the EPRT approximation are solved. Purple: FEM solver (COMSOL), Grey: EPRT solver (Fortran), Green: data treatment steps (Matlab). 
In particular, the updated heat flux $q_{b n}^{\text {new }}$ is obtained at the BTE-HE boundary. Next, $q_{b n}^{\text {new }}$ is introduced as a boundary condition in the HE sub-domain (in conjunction with the conditions at the external boundaries) for solving HE by FEM, which allows finding the temperature and heat flux in the HE subdomain (in our case, this is done by means of the 'COMSOL with Matlab' tool). In particular, this allows obtaining an updated temperature $T_{b}^{\text {new }}$ at the BTE-HE boundary. At this stage, the temperature and heat flux fields of the whole domain have been computed. A first iteration is therefore completed. A convergence criterion is then to be checked at the BTE-HE boundary. $T_{b}^{\text {new }}$ is compared with $T_{b}$, and the same for $q_{b}^{\text {new }}$ and $q_{b}$. If the converging criterion is not satisfied, a new loop is necessary. The process involving the two sub-domains is renewed until convergence is reached.

Some complication can arise at the end of the first iteration, if the initialization (zeroth step) leads to temperature/flux fields too far from the actual solution. At the BTE-HE boundary, temperature may become larger than heat source temperature or smaller than the cold bath one. In this case some condition can be added to limit the temperature variation within a certain range, with a relaxation criterion $T_{b}^{\text {new } 2}=f\left(T_{b}^{\text {new }}, T_{b}\right)$. It was found in particular that this is needed if initialization is done by solving the HE over the whole domain. A simple function $f\left(T_{b}^{\text {new }}, T_{b}\right)=\frac{T_{b}^{\text {new }}+T_{b}}{2}$ was chosen in our case.

Such coupling process was tested and, while initially temperature and flux seemed to converge when iterating and reached distributions close to the actual full EPRT solution after several loops, the process ended up to diverge, generally after the $7^{\text {th }}$ loop. This is in contrast with the $1 \mathrm{D}$ case and with the $2 \mathrm{D}$ case of Vallhabeni et al. ${ }^{21}$, who applied a similar strategy but where the BTE-HE boundary was also a physical boundary between materials leading to a thermal boundary resistance. We will show below that additional consideration was therefore required in our case.

\section{V.B. Additional requirements for coupling and criteria}

Different strategies were tested to control the over-reaction in the coupling process. We note that the starting of the divergence process is best seen when observing the flux distribution (see Fig. 5(b)). The strategies were as follows:

(1) Smoothening the temperature on the boundary. We noted small difference in temperature $T_{b}$ for symmetric points with respect to the vertical axis of symmetry. Ascribing the average to each points did not improve much. 
(a)

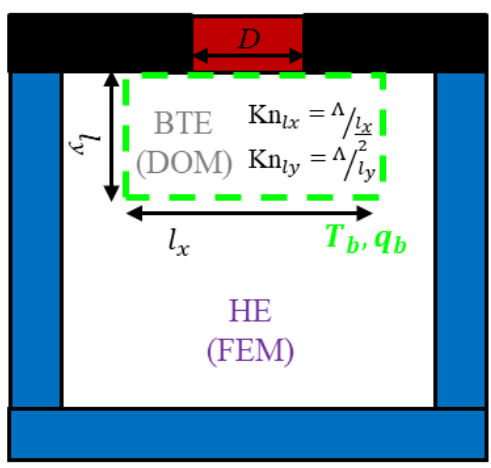

(c)

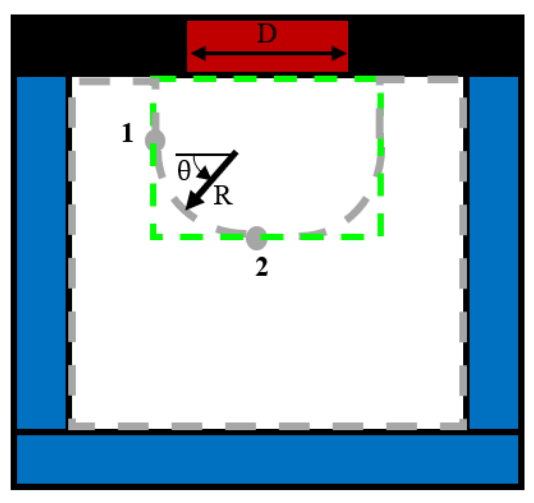

(b)

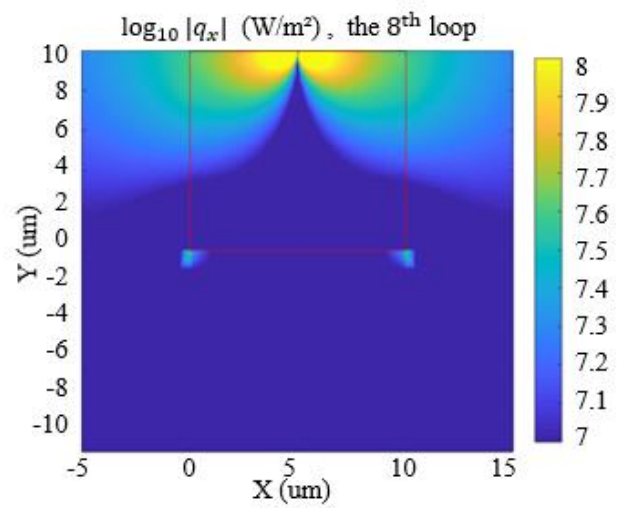

(d)

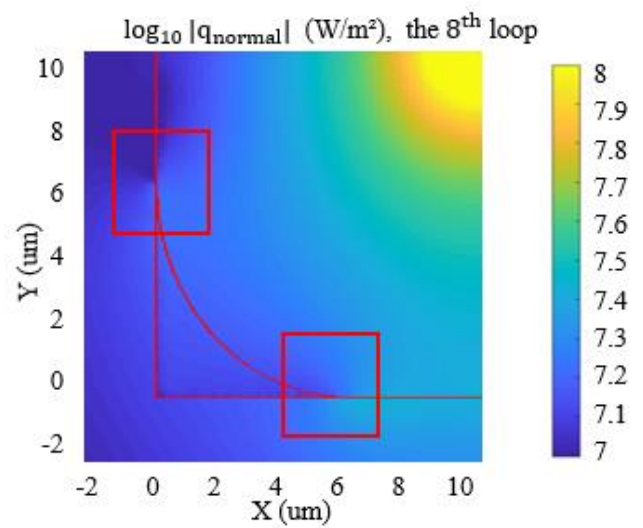

(e)

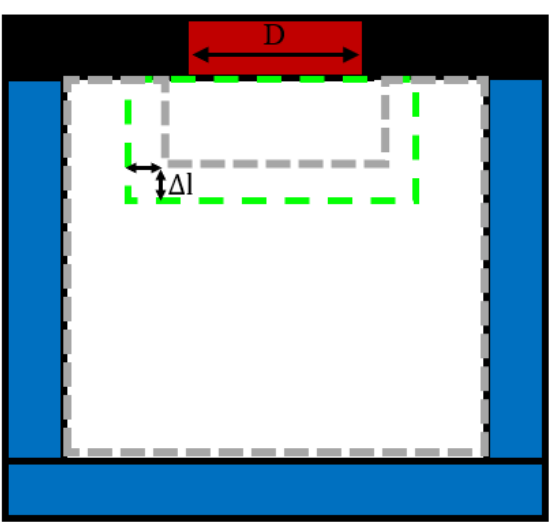

Figure 5. Strategies for improving the convergence. (a) Intuitive coupling strategy, found insufficient. (b) Observation of the flux field at the $8^{\text {th }}$ step, when the divergence process becomes obvious. (c) Strategy of eliminating the abrupt change of boundary condition by rounding the corners. (d) Observation of the flux field at the $8^{\text {th }}$ step, when the difficulty at the connection between the rounded part and the initial boundary is observed. (e) Strategy of overlapping the BTE and HE sub-domains: the 
boundary condition $T_{b}$ for the HE sub domain is taken inside the BTE sub-domain (green dotted rectangle). The overlapping region has the fixed width $\Delta l=0.5 \mu \mathrm{m}$.

(2) Eliminating the corners. Since it was observed that the divergence starts close to the corners (see Fig. 5 (b)), various tests where the corners are replaced by rounded (circular) boundaries for the HE subdomain were implemented (Fig. 5(c)). Quadratic and quartic (continuity of flux and total power crossing the boundary at the circle) laws interpolating between the normal fluxes at the extremities of the circles were tested, but it was observed that the connection between the circles can still lead to issues after few loops (see Fig. 5(d)).

(3) Taking the boundaries for the HE sub-domain inside the BTE domain. Since some issues were always present at the connections points and boundaries between the two sub-domains, it was decided to choose a region inside the BTE domain for the boundary of the HE sub-domain. In this way, some discretization issue at the boundary may be lifted. In the end, it means that the two sub-domains possess a common zone, called the "overlapping zone" and highlighted in Fig. 5(e). It was found that this method allows for convergence of the process.

As a result of this phase of tests, the convergence was found for the relaxation function and the overlapping of BTE and FEM sub-domains displayed in Fig. 5(e), leading to the results of Fig. 6. Convergence is ensured by analyzing the relative difference of heat flux at boundary between current and previous loops $\left|\frac{q_{b}-q_{b}^{\text {new }}}{q_{b}}\right|<\varepsilon_{c}$, where $\varepsilon_{c}$ is the critical value of relative difference and is equal to $10^{-6}$. Such condition is more accurate than a condition on temperature. The heat flux distribution is shown in Fig. 6. A small deviation between the fluxes is observed in Fig. 6(b) when the flux in the overlapping zone is chosen from the DOM results, which is due to a discontinuity of the heat flux in the direction parallel to the interface at the sub-domain boundary. As a consequence, the heat flux should preferentially be chosen in the HE sub-domain in order to keep the connection between the two subdomains smooth (Fig. 6(c)). We note that coupling FEM, which does not inherently guarantee local flux conservation, and Finite-Volume Method may not be the optimum choice from the mathematical side, however it is shown here that a solution for the coupling of popular FEM and DOM/FVM solvers is found.

\section{Performances}


The performances of the coupling process were assessed with respect to two objectives: (i) obtaining accurate results and (ii) obtaining results as fast as possible. This required first to select a given size for the BTE sub-domain and then varying this size.

(a)

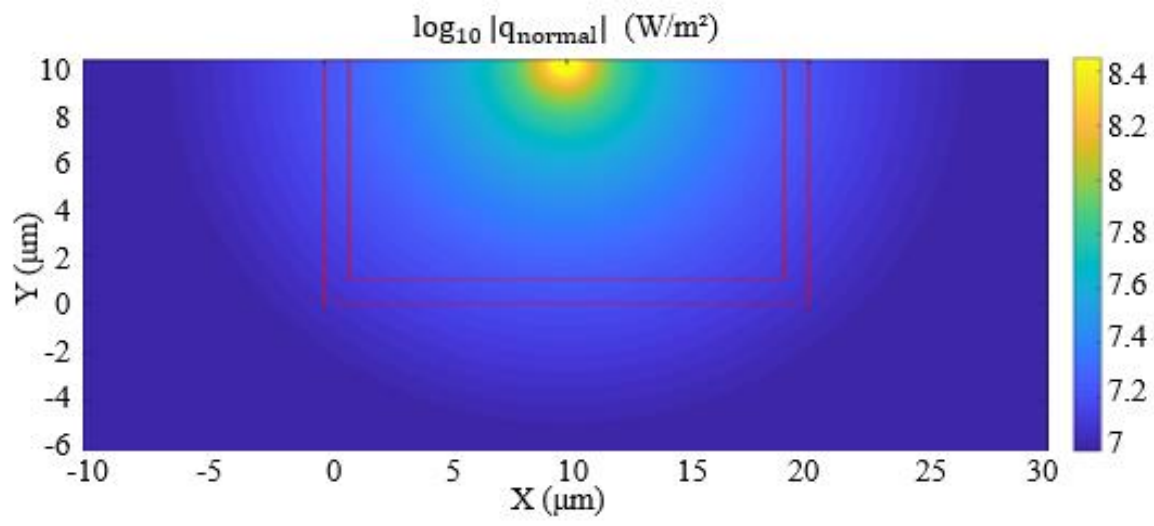

(b)

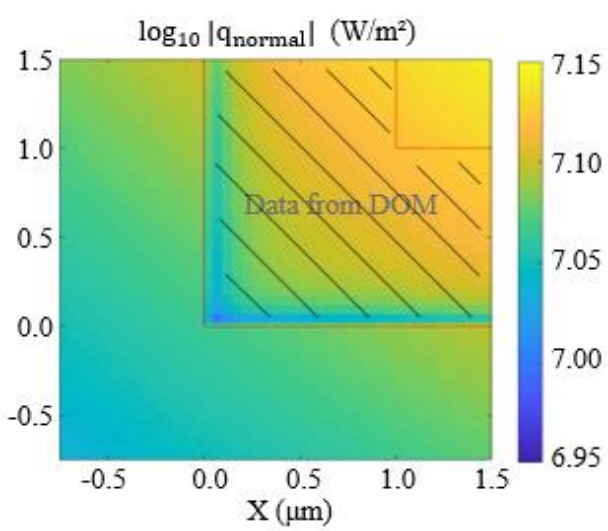

(c)

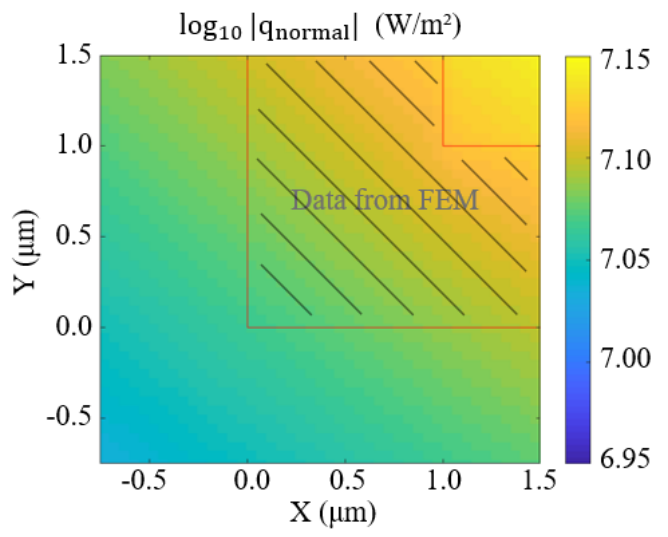

Figure 6. Heat flux field after convergence of the method involving BTE/HE sub-domain coupling. (a) Whole distribution of the normal flux, $(b, c)$ zooms on the connecting boundaries with data in the overlapping region from (b) the EPRT solver and (c) the FEM solver.

VI.A. Efficiency and accuracy of the coupling 
Three initial simulations were performed: two with the coupling process involving different number of mesh elements, and one without coupling where the EPRT is solved by DOM over the whole domain. In the coupling cases, the calculation time of FEM is about seconds and therefore much smaller than that of DOM. Thus, it can be concluded that time is mostly due to the EPRT resolution (in our case by DOM) and it was found that it is linear to the number of mesh elements by comparing tests 1,2 and the reference. The EPRT (DOM) calculation is time consuming and most probably limited by the random access memory, especially if the calculation is performed with desktop computer. As a result, the discretization used for very-large domains cannot be as fine as in the case of small domains. Here, the same mesh size (mesh element size of $0.1 \mu \mathrm{m}$ ) could be kept identical for the reference simulation where the whole domain is solved by DOM (see Tab. 1) and for test case 1, so the heat flux of these two tests could be compared to analyze the accuracy of the coupling method. For all three cases, the tolerance of relative phonon intensity in the DOM computation was kept equal to $10^{-18}$ and the convergence criterion for the coupling process was focused on a relative difference of heat flux set as $\varepsilon_{c}=10^{-6}$ (as mentioned in Section V.B). Tab. 1 shows that despite better resolution close to the heat source the coupling process allows obtaining the results in much faster way, at least by one order of magnitude.

\begin{tabular}{|c|c|c|c|c|}
\hline Test & Method & $\begin{array}{c}\text { Dimension of BTE } \\
\text { DOM } \\
\text { (sub-)domain }\end{array}$ & $\begin{array}{l}\text { Number of mesh elements in } \\
\text { the BTE DOM (sub-)domain }\end{array}$ & $\begin{array}{l}\text { Calculation } \\
\text { time }\end{array}$ \\
\hline 1 & \multirow{2}{*}{$\begin{array}{l}\text { Coupling } \\
\text { process }\end{array}$} & \multirow{2}{*}{$\begin{array}{l}l_{x}=20 \mu \mathrm{m} \\
l_{y}=10 \mu \mathrm{m}\end{array}$} & $n_{x}=200, n_{y}=200$ & $\sim 1$ day \\
\hline 2 & & & $n_{x}=400, n_{y}=400$ & $\sim 4$ days \\
\hline Ref. & $\begin{array}{l}\text { EPRT by DOM } \\
\text { over whole } \\
\text { domain }\end{array}$ & $\begin{array}{l}l_{x}=100 \mu \mathrm{m}, \\
l_{y}=100 \mu \mathrm{m}\end{array}$ & $n_{x}=1000, n_{y}=1000$ & $\sim 4$ weeks \\
\hline
\end{tabular}

Table 1. Parameters of three tests for determining the efficiency and accuracy of the coupling method for comutation over a $(100 \mu \mathrm{m})^{2}$ domain

The relative difference between the results of the reference and case 1 is shown in Fig. 7 by mapping $\left|\frac{q_{\text {coupling }}-q_{E P R T}}{q_{E P R T}}\right|$. We remind that we focus on the flux, which is more sensitive. It is observed that the maximal relative difference takes place at the top sides, where the boundary condition is thermal insulation and therefore $q_{E P R T} \sim q_{\text {coupling }} \sim 0$. The relative difference in the other parts of the domain is at worst $\sim 5 \%$, in particular around the heater. This might be due to the size of the BTE sub-domain selected and is investigated below. Comparing tests 1 and 2, we verified also that an increase of mesh density helps reducing this error, giving an evidence that some error is due to the somehow loose mesh 
close to the thermal source $\left(\frac{\Delta x}{\Lambda} \sim 0.5\right.$ in test 1 and 0.25 in test 2$)$. Note also that the error seems to be large especially around some directions, and could therefore be linked to the angular discretization ('ray effects', see Ref. [36]). We speculate that it could be reduced for an improved order of discretization. In the end, the obtained results with the coupling seem to be close to those with the whole EPRT/DOM solver, which is itself probably not fully accurate due to the compromise needed to reach convergence.

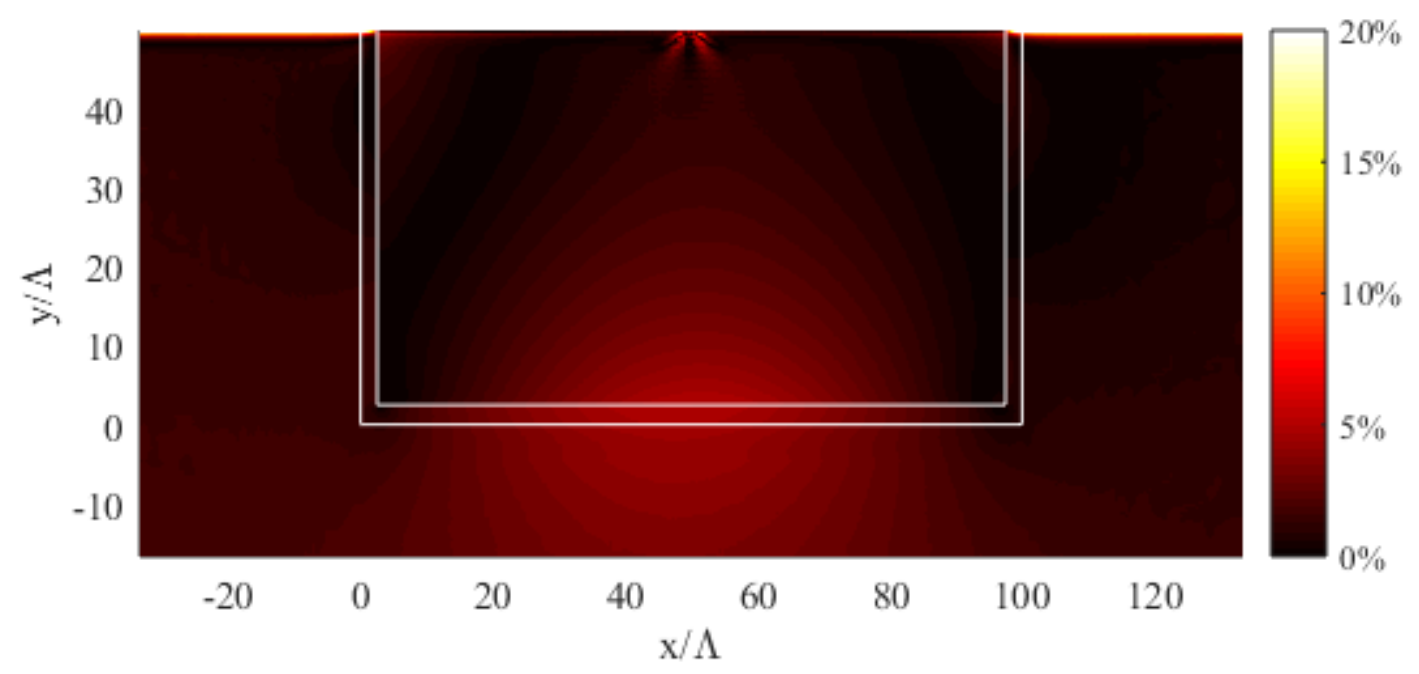

Figure 7. Map of relative difference of heat flux between the coupling and the EPRT. Only DOM domain and a small region close to overlapping area is shown in order to observe more details in DOM domain. White lines indicate the domain boundaries.

VI.B. Optimal size of the BTE sub-domain

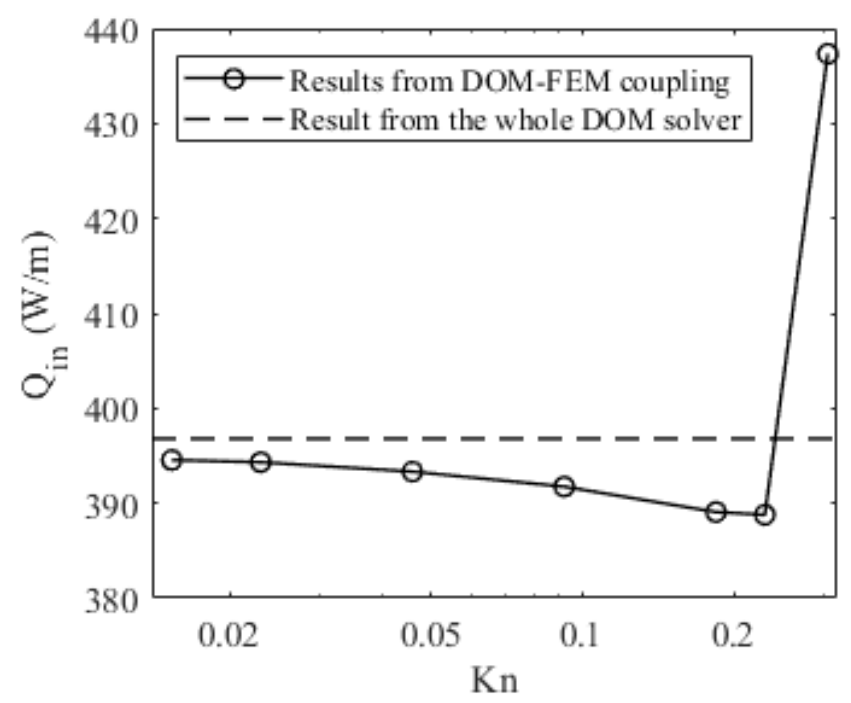

Figure 8. Total heat flux dissipated into the domain as a function of the Knudsen number of the BTE sub-domain (solid line). 
The size of the BTE sub-domain has to be as large as possible to be close to the exact result, but as small as possible for minimizing the computation time. A compromise is therefore to be selected. A Knudsen number can be associated to the BTE sub-domain size (from its largest dimension), and should be as small as possible for improved accuracy. We have varied the dimension of the BTE sub-domain DOM so that the corresponding Knudsen number was changed from 0.3 to 0.017 . The total heat flux into the domain was obtained by computing $Q_{i n}=\oint_{\boldsymbol{q} \cdot \boldsymbol{n}>\mathbf{0}} \boldsymbol{q} \cdot \boldsymbol{n} d l$, where $\boldsymbol{n}$ is unit normal vector of boundary and $d l$ is element of boundary, and is reported in Fig. 8. When the sub-domain Kn decreases, the integrated heat flux into the whole domain converges to a certain value, which is close to the numerical solution of the reference case where the EPRT is solved over the whole domain. It can be concluded that when Kn approaches 0.02 , the relative difference between the coupling process solution and the reference result is smaller than $0.25 \%$. This means that the BTE sub-domain size should allow for diffusive conduction close to its boundary. Indeed, Fig. 1(b) clearly shows that the diffusive regime is achieved for such value of the Knudsen number. Interestingly, even when $\mathrm{Kn}$ is as large as 0.1 in a 2D problem, the relative difference between the two solutions stays below $1 \%$ as shown in Fig. 8. Hence, it is possible to chose $\mathrm{Kn}=0.1$ for the effective Knudsen number of the BTE sub-domain in a $2 \mathrm{D}$ configuration and therefore save time while the error stays limited.

\section{Conclusions and prospects}

The semi-classical Boltzmann transport equation, here solved within the EPRT approximation, has the capacity to correctly describe heat conduction by phonon in both diffusive and ballistic regime. However, calculation time increases exponentially while Kn decreases from ballistic domain to diffusive domain, which is a strong inconvenience. We have introduced a simple method to solve efficiently phonon heat conduction over large domains, which is based on the coupling between the Boltzmann equation (solved by DOM with a Finite Volume Method for the spatial discretization) and the heat equation (solved with FEM). It aims at saving simulation time while maintaining the accuracy of the results. From all the numerical simulations above and the hypotheses used in this report, several conclusions can be drawn:

1. Coupling the BTE and the heat equation allows reducing the computational time. This was realized with an iterative method, which involves an overlap between the sub-domains for configurations beyond $1 \mathrm{D}^{22}$ and when no thermal boundary between differing materials is present ${ }^{21}$. The coupling method helps reducing the total computational time by an order of magnitude (factor $\sim 5$ or $\sim 20$ depending on the accuracy expected from the results), demonstrating the feasibility of this new method.

2. The performance of the coupling has been investigated. For selected sets of parameters, the error stays limited below $5 \%$ away from insulating boundaries. 
3. The effective Knudsen number required for the BTE sub-domain has been determined. A Knudsen number equal to 0.02 is required to ensure diffusive conduction but numerical results show that values up to 0.1 are still acceptable. This allows to further reduce the computational time by an order of magnitude.

A few comments are in order now.

(i) Here, only a single mean free path was used. However, a spectral BTE can also be used in the BTE sub-domain, and the present results are providing insights toward such approach. Convergence would certainly be achieved for BTE sub-domain size designed as a function of the largest mean free path. Faster results would be obtained by selecting the size of such domain from the largest significantlycontributing mean free path. It may happen for some materials that these large mean free paths have sizes close to that of the whole domain and that their contribution to the total energy transfer is significant ${ }^{37}$, precluding the use of the method highlighted here. In this case, additional strategies for addressing short MFPs without solving the BTE would be the solution for reducing the computational time ${ }^{19-21}$.

(ii) We anticipate universal application of the method, which was tested only with DOM and FEM, to other types of solvers. It is possible that the overlap would be not required for solvers based on different schemes. For the phonon BTE, the Monte Carlo method is spreading due to its ability to cope with very complex geometry. The statistical and random error nature of the MC results could lead to additional complexity, requiring regularization at the boundary ${ }^{38}$ or extra thermostat ${ }^{22}$.

(iii) While the coupling was only tested in Cartesian 2D geometry, it is certainly possible to generalise to $3 \mathrm{D}$ as was shown by Vallabhaneni et al. ${ }^{21}$ in the particular case of a GaN transistor.

(iv) Implementing the coupling process in configurations where amorphous materials, thermal boundary resistances or electronic heat conduction are present will open a full avenue for quick and accurate thermal simulations.

There are certainly many more strategies possible for improving the speed of phonon multiscale heat conduction, in particular adaptive meshes in the BTE domains. However, as modern technology has stepped into nanoscale and exact solutions for multiscale thermal problems have become an urgent challenge for industry ${ }^{39,40}$, the present coupling method does already provide a useful tool for current demand and has broad and easy application prospects.

\section{Acknowledgements}

The financial supports of EU projects QuantiHeat and EFINED and French ANR project TIPTOP are gratefully acknowledged. Early discussions with T. Nghiem and J. Randrianalisoa is acknowledged. We 
thank E. Nefzaoui and D. Lemmonier for discussions and providing numerical codes. We thank also anonymous referees for useful suggestions.

\section{References}

1. Ziman, J. Electrons and Phonons. The theory of transport phenomena in solids. (Oxford University Press, 1961).

2. Collective. Microscale and Nanoscale Heat Transfer - Topics in Applied Physics 107. (Springer Verlag, 2007).

3. Chapuis, P. O. Chapter 3: Introduction to heat transfer at the nanoscale. in Thermometry at the Nanoscale: Techniques and Selected Applications (eds. Carlos, L. D. \& Palacio, F.) (RSC Publishing, 2016). doi:10.1039/9781782622031-00039.

4. Chen, G. Nanoscale Energy Transport and Conversion. (Oxford Engineering Science Series/MIT-Pappalardo Series in Mechanical Engineering, 2005).

5. Ju, Y. S. \& Goodson, K. E. Phonon scattering in silicon films with thickness of order $100 \mathrm{~nm}$. Appl. Phys. Lett. 74, 3005 (1999).

6. Hamian, S., Yamada, T., Faghri, M. \& Park, K. Finite element analysis of transient ballisticdiffusive phonon heat transport in two-dimensional domains. Int. J. Heat Mass Transf. 80, 781788 (2015).

7. Lacroix, D., Joulain, K. \& Lemonnier, D. Monte Carlo transient phonon transport in silicon and germanium at nanoscales. Phys. Rev. B 72, 64305 (2005).

8. Randrianalisoa, J. \& Baillis, D. Monte Carlo Simulation of Steady-State Microscale Phonon Heat Transport. J. Heat Transfer 130, 072404 (2008).

9. Wong, B. T., Francoeur, M. \& Pinar Mengüç, M. A Monte Carlo simulation for phonon transport within silicon structures at nanoscales with heat generation. Int. J. Heat Mass Transf. 54, 18251838 (2011).

10. Murthy, J. et al. Review of Multiscale Simulation in Submicron Heat Transfer. Int. J. Multiscale Comput. Eng. 3, 5-32 (2005).

11. Mittal, A. \& Mazumder, S. Generalized Ballistic-Diffusive Formulation and Hybrid SN-PN Solution of the Boltzmann Transport Equation for Phonons for Nonequilibrium Heat Conduction. J. Heat Transfer 133, 92402 (2011).

12. Mittal, A. \& Mazumder, S. Hybrid discrete ordinates - spherical harmonics solution to the Boltzmann Transport Equation for phonons for non-equilibrium heat conduction. J. Comput. Phys. 230, 6977-7001 (2011).

13. Volz, S., Saulnier, J.-B. \& Lemonnier, D. Clamped nanowire thermal conductivity based on phonon transport equation. Microscale Thermophys. Eng. 5, 191-207 (2001). 
14. Terris, D., Joulain, K., Lemonnier, D. \& Lacroix, D. Modeling semiconductor nanostructures thermal properties: The dispersion role. J. Appl. Phys. 105, 73516 (2009).

15. Majumdar, A. Microscale Heat Conduction in Dielectric Thin Films. J. Heat Transfer 115, 7-16 (1993).

16. Chen, G. Ballistic-Diffusive Heat-Conduction Equations. Phys. Rev. Lett. 86, 2297-2300 (2001).

17. Zhang, C., Chen, S. \& Guo, Z. An implicit kinetic scheme for multiscale heat transfer problem accounting for phonon dispersion and polarization. Int. J. Heat Mass Transf. 1366-1376 (2019).

18. Luo, X.-P., Zhao, Y.-F. \& Yi, H.-L. Multiscale phonon transport simulations by a discrete ordinate method with streaming and collision processes. Numer. Heat Transf. Part A Appl. 75, 381-401 (2019).

19. Loy, J. M., Murthy, J. Y. \& Singh, D. A Fast Hybrid Fourier-Boltzmann Transport Equation Solver for Nongray Phonon Transport. J. Heat Transfer 135, 011008 (2012).

20. Allu, P. \& Mazumder, S. Hybrid ballistic-diffusive solution to the frequency-dependent phonon Boltzmann Transport Equation. Int. J. Heat Mass Transf. 100, 165-177 (2016).

21. Vallabhaneni, A. K., Chen, L., Gupta, M. P. \& Kumar, S. Solving Nongray Boltzmann Transport Equation in Gallium Nitride. J. Heat Transfer 139, 102701 (2017).

22. Li, H.-L., Hua, Y.-C. \& Cao, B.-Y. A hybrid phonon Monte Carlo-diffusion method for ballisticdiffusive heat conduction in nano- and micro- structures. Int. J. Heat Mass Transf. 127, 10141022 (2018).

23. Yang, R., Chen, G., Laroche, M. \& Taur, Y. Simulation of nanoscale multidimensional transient heat conduction problems using ballistic-diffusive equations and phonon Boltzmann equation. $J$. Heat Transfer 127, 298-306 (2005).

24. Modest, M. F. Radiative heat transfer, 3rd edition. Radiative Heat Transfer, 3rd Edition (Academic Press, 2013). doi:https://doi.org/10.1016/C2010-0-65874-3.

25. Lemonnier, D. Solution of the Boltzmann Equation for Phonon Transport. in Microscale and Nanoscale Heat Transfer - Topics in Applied Physics 107 (ed. Volz, S.) 77-106 (Springer Verlag, 2007).

26. Carlson B.G., L. K. D. Transport theory-The method of discrete ordinates. in Computing Methods in Reactor Physics (Gordon and Breach, 1968).

27. Weizheng, C. Thermal dissipation at the nanoscale and impact on mechanical stress. (Ecole Centrale Nantes, 2019).

28. Chapuis, P. O., Nghiem, T. T. T., Da Cruz, C. A. \& Nefzaoui, E. Thermal transport phenomena beyond the diffusive regime. in Proceedings of the 23rd International Conference Mixed Design of Integrated Circuits and Systems, MIXDES 2016 (2016). doi:10.1109/MIXDES.2016.7529695.

29. Siemens, M. E. et al. Quasi-ballistic thermal transport from nanoscale interfaces observed using ultrafast coherent soft X-ray beams. Nat. Mater. 9, 26-30 (2010).

30. Jaber, W. Phonon heat conduction probed by means of an electro-thermal method involving 
deposited micro and nanowires. (INSA Lyon, 2016).

31. Ziabari, A. et al. Full-field thermal imaging of quasiballistic crosstalk reduction in nanoscale devices. Nat. Commun. 9, 255 (2018).

32. Minnich, A. J. Multidimensional quasiballistic thermal transport in transient grating spectroscopy. Phys. Rev. B 92, 85203 (2015).

33. Hu, Y., Zeng, L., Minnich, A. J., Dresselhaus, M. S. \& Chen, G. Spectral mapping of thermal conductivity through nanoscale ballistic transport. Nat. Nanotechnol. 10, 701-706 (2015).

34. Jaber, W. \& Chapuis, P. O. Non-idealities in the $3 \omega$ method for thermal characterization in the low- and high-frequency regimes. AIP Adv. 8, 045111 (2018).

35. Wexler, G. The size effect and the non-local Boltzmann transport equation in orifice and disk geometry. Proc. Phys. Soc. 89, 927-941 (1966).

36. Chai, J. C., Lee, H. S. \& Patankar, S. V. Ray effect and false scattering in the discrete ordinates method. Numer. Heat Transf. Part B Fundam. 24, 373-389 (1993).

37. Moore, A. L. \& Shi, L. Emerging challenges and materials for thermal management of electronics. Mater. Today 17, 163-174 (2014).

38. Nghiem, T. T. T. \& Randrianalisoa, J. Private communication.

39. Blish, R., Durrant, N. \& (eds). Semiconductor Device Reliability Failure Models. (2000).

40. Pop, E. Energy dissipation and transport in nanoscale devices. Nano Res. 3, 147-169 (2010). 